\section{Efeito do treinamento resistido progressivo nos parâmetros antropométricos de pessoas vivendo com HIV/AIDS}

\author{
Effect of progressive resistance training on \\ anthropometric parameters of people living \\ with HIV / AIDS
}

Petrus Gantois Massa Dias dos Santos ${ }^{1}$

Gledson Tavares de Amorim Oliveira ${ }^{1}$

Paulo Roberto Cavalcanti Carvalho ${ }^{1}$

\section{Resumo}

Objetivo: Verificar o efeito do treinamento resistido progressivo no perfil antropométrico de pessoas vivendo com HIV/AIDS, submetidos à terapia anti-retroviral altamente ativa. Metodologia: A amostra foi composta por 30 sujeitos, no qual apenas 18 completaram o protocolo de treinamento. Foi avaliado o índice de massa corporal, a relação cintura/quadril, índice de conicidade, percentual de gordura, massa gorda e magra. $\mathrm{O}$ programa de treinamento resistido progressivo foi constituído por três etapas: 1) envolveu seis sessões adaptativas; 2) englobou 12 sessões com três séries de 12 repetições e intervalo de descanso de 60 segundos(s), com intensidade de $50 \%$ da carga máxima; e 3) constituída por 42 sessões com três séries de oito repetições e intervalo de descanso de 90 segundos(s), com intensidade de 70-80\% da carga máxima. Resultados: Foi verificado redução nas dobras cutâneas subescapular ( $\mathrm{p}=0,047)$, supra-ilíaca $(p=0,029)$ e triciptal $(p=0,018)$ e na circunferência da cintura $(p=0,003)$; na relação cintura/quadril $(p=0,050)$; percentual de gordura $(p=0,040)$ e aumento na circunferência do braço $(p=0,010)$ e da perna $(p=0,005)$ e na massa magra $(p=0,004)$. Conclusões: $O$ presente estudo sugere que a utilização do treinamento resistido progressivo apresentou melhorias nos parâmetros antropométricos associados ao uso da terapia anti-retroviral altamente ativa das pessoas vivendo com HIV/AIDS.

\section{Palavras-chave}

Antropometria; Composição corporal; Terapia em saúde.

\begin{abstract}
Objective: To investigate the effect of progressive resistance training on anthropometric profile of people living with HIVIAIDS, undergoing highly active antiretroviral therapy. Methods: The sample included 30 subjects, and only 18 completed the training protocol. The body mass index, waist/hip ratio, conicity index, body fat percentage and body fat and lean mass were assessed. The progressive resistance training program consisted of three stages: 1) six adaptive sessions 2) 12 sessions with three sets of 12 repetitions and rest interval of 60 seconds (s), at an intensity of $50 \%$ of the maximum load, and 3) 42 sessions with three sets of eight repetitions and 90 seconds rest interval (s), with an intensity of 70-80\% of maximum load. Results: A reduction in skinfold thickness (subscapular $p=0.047$; suprailiac $p=0.029$; and trice $p$ s $p=0.018)$, waist circumference $(p=0.003)$, the waist $/$ hip ratio $(p=0.050)$ and fat percentage $(p=0.040)$, and increase in arm $(p=0.010)$ and leg $(p=0.040)$ circumferences and lean mass ( $p=0.004)$ was observed. Conclusions: This study suggests that the use of progressive resistance training combined with the use of highly active antiretroviral therapy of people living with HIVI AIDS resulted in improvements in anthropometric parameters.
\end{abstract}

\section{Keywords}

Anthropometry; Body composition; Health therapy.
Rev Bras Ativ Fis Saúde p. 782-788

DOI:

http://dx.doi.org/10.12820/rbafs.v.18n6p782

1 Universidade Federal de Pernambuco, Departamento de Educação Física, Recife, Pernambuco, Brasil. 


\section{INTRODUÇÃO}

A Síndrome da Imunodeficiência Adquirida (AIDS) é uma doença crônica, caracterizada por profunda imunossupressão, gerando quadros clínicos como: hipotrofia muscular, degeneração do sistema nervoso central, processos malignos e infecções oportunistas ${ }^{1}$. O vírus da imunodeficiência humana (HIV), mundialmente disseminado, é o agente responsável pela $\mathrm{AIDS}^{2}$.

De acordo com os dados da Joint United Nations Programme on HIV/AIDS 3 , no final de 2011, estimou-se que 34 milhões da população mundial viviam com HIV/AIDS com índice de mortalidade de 1,7 milhões pessoas, havendo maior incidência de casos e mortes na África Subsaariana. No Brasil, de acordo com a mesma entidade supracitada, estima-se que 490.000 pessoas viviam com HIV/ AIDS, sendo considerado o país da America Latina com maior número de pessoas infectadas e que destas 15.000 morreram de causas relacionadas à AIDS.

Após o uso do tratamento da terapia anti-retroviral altamente ativa (HAART) foram observadas mudanças no curso da infecção das pessoas com o vírus da imunodeficiência (HIV), possibilitando uma sobrevida e redução nas taxas de morbidade e mortalidade e consequentemente promovendo um melhor bem-estar das pessoas vivendo com HIV/AIDS ${ }^{4-6}$. Entretanto, de acordo com Santos et al. ${ }^{7}$ o HAART não obteve êxito em eliminar a infecção por HIV, havendo desta forma, a necessidade de uma manutenção a longo prazo deste tratamento para o controle da carga viral. Como efeito da longa exposição ao HAART diversos estudos têm evidenciado uma associação entre o uso dos anti-retrovirais com uma síndrome de redistribuição da gordura corporal, denominada síndrome lipodistrófica ${ }^{2,4,8}$.

Além da síndrome lipodistrófica ocasionada pela utilização dos medicamentos anti-retrovirais, outra alteração prejudicial às pessoas vivendo com HIV/AIDS e decorrentes da infecção é a progressiva redução da massa corporal magra, a qual se encontra associada com a redução da capacidade do músculo-esquelético em gerar força ${ }^{9}$. Diante destas complicações, orientações recentes recomendam a utilização da prática de atividade física como tratamento não farmacológico para as pessoas que vivem com HIV/AIDS ${ }^{10}$.

Alguns estudos têm demonstrado os benefícios dos exercícios resistidos no combate aos efeitos colaterais decorrentes do HAART e da infecção pelo HIV, como: redução da perda de massa magra (maior hipertrofia) e da funcionalidade do músculo (capacidade de gerar força); efeitos positivos na composição corporal em contraste com a lipodistrofia, aumentar a sensibilidade à insulina e melhoria no perfil lipídico e na qualidade de vida ${ }^{4,7,9-11}$. Desta forma, se faz necessário investigar os benefícios decorrentes do exercício resistido nas pessoas vivendo com HIV/ AIDS, para se obter um maior controle das alterações antropométricas e funcionais desta população. Portanto, o objetivo do presente estudo foi verificar o efeito do treinamento resistido progressivo (TRP) no perfil antropométrico de pessoas vivendo com HIV/AIDS, submetidos ao HAART.

\section{MÉTODOS}

\section{Tipo de estudo e amostra}

A presente pesquisa é um estudo do tipo transversal com amostragem intencional, realizada no Departamento de Educação Física da Universidade Federal de Pernambuco no Recife/PE, sendo os sujeitos participantes do projeto "A importância de tratamentos multidisciplinares e sua relação com a melhoria da qualidade de 
vida em adultos vivendo com HIV/AIDS, em e não utilização de terapia anti-retroviral de alta atividade".

A amostra foi composta por 30 pessoas vivendo com HIV/AIDS submetidos ao HAART, todos sem impedimentos físicos. Todos os sujeitos que participaram do estudo assinaram um Termo de Consentimento Livre e Esclarecido (TCLE), de acordo com as normas estabelecidas pela resolução 196/96 do Conselho Nacional de Saúde para pesquisas envolvendo seres humanos, com critérios de inclusão considerados indivíduos que não possuíam qualquer enfermidade e/ou limitações físicas que inviabilizasse a realização dos exercícios. O estudo foi aprovado pelo Comitê de Ética do Centro de Ciências da Saúde, da Universidade Federal de Pernambuco (processo 262/11).

\section{Avaliações antropométricas}

Foi mensurada a massa corporal (kg) em uma balança portátil (Filizola) com resolução próxima de $0,1 \mathrm{~kg}$, a estatura corporal através de uma estadiômetro (Sanny), com resolução próxima de $0,1 \mathrm{~cm}$, (a fim de se obter o Índice de Massa Corporal - IMC), bem como as circunferências da cintura (CC), do quadril (CQ) e do abdômen (CA), através de uma fita antropométrica não flexível (Sanny) para a estimação da Relação Cintura/Quadril (RCQ) e do Índice de Conicidade (IC). Para calcular a densidade corporal foi utilizado o protocolo de Durnin e Womersley ${ }^{12,13}$, estimado pelo somatório de quatro dobras cutâneas (bíceps, tríceps, subescapular e supra-ilíaca), por meio de um adipômetro Cescorf, com precisão de $1 \mathrm{~mm}$. Para a identificação do percentual de gordura recorreu-se a equação de Siri ${ }^{14}$.

As mensurações antropométricas foram realizadas no momento pré-treino e ao final das sessões de treinamento. Para efeito deste estudo, todas as medidas seguiram as normas estabelecidas pela ISAK ${ }^{15}$. A fim de verificar a fidedignidade da avaliação foram realizados o erro técnico de medida $(<5 \%)^{16}$ e o coeficiente de teste reteste para todos os pontos anatômicos, obtendo valores acima de r=0,95.

\section{Protocolo de treinamento}

O protocolo de TRP foi constituído por 60 sessões, sendo dividido em três etapas (1-preparatória, 2-transitória e 3-especifica) ${ }^{7}$. A etapa inicial foi constituída por seis sessões, a qual envolveu o período de adaptação, visando esclarecer a correta execução dos exercícios, com a intensidade sendo estabelecida através da escala de Borg, a qual se manteve entre leve e moderada ${ }^{17}$. Após o período adaptativo todos os sujeitos da pesquisa realizaram um teste submáximo de 10 repetições máximas $(\mathrm{RM})^{18}$, para predizer a carga relativa a $1 \mathrm{RM}$. A segunda etapa englobou 12 sessões com três séries de 12 repetições e intervalo de descanso de 60 segundos(s), com intensidade de 50\% do RM e velocidade de execução de 2121 (2s de contração concêntrica para 1s de descanso e $2 \mathrm{~s}$ de contração excêntrica para $1 \mathrm{~s}$ de descanso). A fase especifica envolveu as 42 sessões finais, sendo constituída por três séries de oito repetições com intervalo de descanso de 90 s, intensidade de 70-80\% do RM e velocidade de execução de 2020. O protocolo de TRP seguiu as recomendações e os princípios básicos do treinamento e prescrição do exercício físico proposto pela American College of Sport Medicine ${ }^{19}$.

$\mathrm{O}$ método do treinamento utilizado foi o alternado por segmento, sendo dividido em treinos 'A' e 'B', no quais cada sessão envolveu seis exercícios. O treino 'A' foi composto pelos seguintes exercícios e ordem: supino vertical, leg press horizontal, tríceps no pulley, cadeira extensora, desenvolvimento e cadeira abdutora já o treino 'B' foi constituído pelos exercícios: puxada frontal, cadeira flexora, rosca 
bíceps direta, panturrilha no leg press, rosca punho supinada e glúteo na máquina. Todo o procedimento experimental foi supervisionado por dois professores e três graduandos de Educação Física, previamente treinados.

\section{Análise estatística}

Os dados foram reportados através da análise descritiva. Para verificar a normalidade dos dados foi realizado o teste de Shapiro-Wilk. A comparação entre o período pré e pós-treinamento resistido nas variáveis antropométricas das pessoas vivendo com HIV/AIDS foi realizada através do teste $t$ para amostra pareada, sendo que os dados estão apresentados como médias e desvios-padrão. O nível de significância estabelecido foi de $\mathrm{p}<0,05$. A análise estatística foi realizada através do software Statistical Package for the Social Sciences - SPSS versão 17.0.

\section{RESULTADOS}

Da amostra inicial, apenas 18 sujeitos finalizaram o protocolo de treinamento, havendo uma perda amostra de $40 \%$, por motivos de adaptabilidade ao treinamento, ausência de condições para a locomoção ao local do estudo, agravamento no quadro clínico e por motivos pessoais. Desta forma, a amostra foi composta por 10 sujeitos do sexo masculino (55,6\%), com idade média de 38,9 $\pm 8,22$ anos e submetidos a 8,61 \pm 6,64 anos ao HAART.

A tabela 1 apresenta os resultados dos efeitos das 60 sessões do TRP nas variáveis antropométricas das pessoas vivendo com HIV/AIDS, os quais apresentaram maiores valores pós-treino para a circunferência do braço $(\mathrm{p}=0,010)$ e da panturrilha $(\mathrm{p}=0,005)$ e menores valores para as dobras cutâneas subescapular $(\mathrm{p}=0,047)$, supra-ilíaca $(\mathrm{p}=0,029)$, triciptal $(\mathrm{p}=0,018)$ e para a circunferência da cintura $(p=0,003)$.

Tabela 1 - Variáveis antropométricas das pessoas vivendo com HIV/AIDS antes (pré) e após (pós) 60 sessões de treinamento resistido progressivo.

\begin{tabular}{lccc}
\hline \multicolumn{1}{c}{ Variáveis } & Pré-treino & Pós-treino & p \\
\hline Massa corporal $(\mathrm{kg})$ & $66,87 \pm 10,07$ & $67,96 \pm 10,61$ & 0,228 \\
\hline D.C subescapular $(\mathrm{mm})$ & $16,27 \pm 6,72$ & $13,67 \pm 4,67$ & 0,047 \\
\hline D.C supra-ilíaca $(\mathrm{mm})$ & $13,72 \pm 6,75$ & $12,17 \pm 5,93$ & 0,029 \\
\hline D.C bicipital $(\mathrm{mm})$ & $7,08 \pm 3,62$ & $6,76 \pm 3,36$ & 0,440 \\
\hline D.C tricipital $(\mathrm{mm})$ & $14,79 \pm 8,06$ & $12,72 \pm 7,61$ & 0,018 \\
\hline Circ. cintura $(\mathrm{cm})$ & $83,34 \pm 8,93$ & $80,78 \pm 8,32$ & 0,003 \\
\hline Circ. quadril $(\mathrm{cm})$ & $95,22 \pm 9,54$ & $92,91 \pm 11,16$ & 0,112 \\
\hline Circ. braço $(\mathrm{cm})$ & $28,41 \pm 2,30$ & $29,33 \pm 2,46$ & 0,010 \\
\hline Circ. panturrilha $(\mathrm{cm})$ & $35,60 \pm 3,14$ & $36,74 \pm 3,20$ & 0,005 \\
\hline
\end{tabular}

D.C = dobra cutânea; Circ.=circunferência.

A tabela 2 ilustra o efeito do TRP na composição corporal das pessoas vivendo com HIV/AIDS. Após as 60 sessões evidenciou-se uma redução na RCQ $(\mathrm{p}=0,050)$ e no percentual de gordura $(\mathrm{p}=0,040)$ e um aumento na massa magra $(\mathrm{p}=0,004)$. 
Tabela 2. Composição corporal das pessoas vivendo com HIV/AIDS antes (pré) e após (pós) 60 sessões de treinamento resistido progressivo.

\begin{tabular}{lccc}
\hline Composição Corporal & Pré-treino & Pós-treino & p \\
\hline IMC $\left(\mathrm{kg} / \mathrm{m}^{2}\right)$ & $24,97 \pm 4,41$ & $25,56 \pm 4,62$ & 0,215 \\
\hline Relação Cintura/Quadril & $0,90 \pm 0,12$ & $0,87 \pm 0,09$ & 0,050 \\
\hline Índice de conicidade & $1,25 \pm 0,14$ & $1,24 \pm 0,16$ & 0,768 \\
\hline Percentual de Gordura (\%) & $27,25 \pm 6,24$ & $25,71 \pm 5,86$ & 0,040 \\
\hline Massa magra $(\mathrm{kg})$ & $48,37 \pm 6,38$ & $50,11 \pm 6,11$ & 0,004 \\
\hline Massa gorda $(\mathrm{kg})$ & $18,50 \pm 6,04$ & $17,85 \pm 6,28$ & 0,288 \\
\hline
\end{tabular}

IMC= índice de massa corporal.

\section{DISCUSSÃO}

$\mathrm{Na}$ literatura alguns estudos relatam que o treinamento resistido proporciona melhorias no peso, resultantes de aumento na massa magra e redução no percentual de gordura e nas circunferências da cintura e abdômen ${ }^{7,9,11}$. Além das mudanças na composição corporal, Yarasheski et al. ${ }^{20}$, complementam que o treinamento resistido aumenta a área de secção do músculo, o que proporciona maiores benefícios na força muscular e acarreta a preservação da massa muscular promovendo a manutenção das reservas energéticas, favorecendo o funcionamento das funções vitais do indivíduo ${ }^{21}$.

Após as 60 sessões de TRP observaram-se nas variáveis antropométricas reduções da gordura corporal e aumento da massa magra em diferentes segmentos corporais. Em relação às variáveis antropométricas verificou-se redução nos seguintes segmentos: dobras cutâneas subescapular, supra-ilíaca e tricipital e na circunferência da cintura. Estes dados se apresentaram de forma semelhante ao estudo de Santos et al. ${ }^{22}$, que realizaram uma intervenção de 60 sessões de TRP em 06 pacientes com HIV/AIDS em Ribeirão Preto, São Paulo, e verificaram redução média para as variáveis supracitadas. Segundo os mesmos autores, esta área se caracteriza pela lipohipertrofia causada pela utilização da HAART ${ }^{9}$.

Além destes achados, o presente estudo apontou para um aumento das circunferências do braço e da perna. Estudo realizado por Jones et al. ${ }^{23}$, ao investigarem seis pacientes com HIV/AIDS, também verificaram aumento na circunferência do braço e da perna. Entretanto, vale ressaltar que os mesmos realizaram 10 semanas de treinamento concorrente.

No que concerne à composição corporal dos pacientes vivendo com HIV/ AIDS, estudos apontam para uma similaridade com os nossos achados. Brito et al. ${ }^{9}$ ao investigarem a influência de 24 semanas de treinamento resistido em indivíduos infectados pelo HIV/AIDS divididos em grupo experimental ( $\mathrm{n}=23$ ) e controle ( $n=22)$, encontraram redução no percentual de gordura somente no grupo experimental. Estudo realizado por Guerra ${ }^{24}$ com indivíduos vivendo com HIV/ AIDS ( $n=15$ ) identificou após 16 semanas de treinamento resistido redução no percentual de gordura. $\mathrm{O}$ aumento obtido nesse estudo para a massa magra gerou equivalência aos achados de Santos et al. ${ }^{7}$ com pacientes com HIV/AIDS submetidos a 36 sessões de TRP, quando os autores verificaram aumento para a massa magra de 1,85 $\pm 1,69 \mathrm{~kg}$. Roubenoff et al. ${ }^{25}$, ao realizarem um programa de TRP três vezes por semana de forma não consecutiva constituído por oito semanas de duração, verificaram redução da massa gorda em concomitância com o aumento na massa magra após realização de oito semanas. Yarasheski et al..$^{20}$ ao realizarem 16 semanas (quatro sessões por semana), verificaram aumento na área de secção mus- 
cular, na massa magra e força muscular. Estes mesmos autores ainda enfatizaram a realização do treinamento resistido como uma intervenção efetiva para o aumento da força muscular e massa magra em pacientes com HIV/AIDS.

Como principal limitação do presente estudo pode-se citar a ausência de um grupo controle, no qual possibilitaria maiores comparações sobre o efeito do TRP nos parâmetros antropométrico de pessoas vivendo com HIV/AIDS submetidos ao HAART. No entanto, vale ressaltar que esse estudo se torna um dos poucos realizados no Brasil, mais especificamente na região Nordeste, evolvendo um acompanhamento de 60 sessões TRP com a presente amostra.

\section{CONCLUSÃO}

O presente estudo sugere que a utilização do TRP apresentou melhorias nos parâmetros antropométricos associados ao uso do HAART das pessoas vivendo com HIV/AIDS. De forma geral, os resultados apontam para a redução na gordura corporal e aumento da massa magra, o que pode se tornar um importante fator de desfecho para a saúde destes, afetando, consequentemente, sua qualidade de vida.

\section{Contribuiç̧ão dos autores}

Petrus Gantois Massa Dias dos Santos, Gledson Tavares de Amorim Oliveira e Paulo Roberto Cavalcanti Carvalho participaram desde a elaboração do projeto à redação do artigo.

\section{REFERÊNCIAS}

1. Souza HF, Marques DC. Benefícios do treinamento aeróbio e/ou resistido em indivíduos HIV+: Uma Revisão Sistemática. Rev Bras Med Esporte. 2009; 15: 467-71.

2. Brito CJ, Mendes EL, Bastos AA, Nóbrega OT, Paula SO, Córdova C. O papel do exercício na era da terapia anti-retroviaral fortemente ativa. Rev. Bras. Ci. Mov. 2010; 18: 109-16.

3. UNAIDS. Global report: UNAIDS report on the global AIDS epidemic 2012. Disponível em: <http://www.unaids.org>. Acesso em: 10/09/2013.

4. Mendes EL, Andaki ACR, Amorim PRS, Natali AJ, Brito CJ. Treinamento físico para indivíduos HIV positivo submetidos a HAART: efeitos sobre parâmetros antropométricos e funcionais. Rev Bras Med Esporte 2013; 19: 16-21.

5. Gomes Neto M, Ogalha C, Andrade AM, Brites C. A systematic review of effects of concurrent strength and endurance training on the health-related quality of life and cardiopulmonary status in patients with HIV/AIDS. BioMed Res Int. 2013; 2013; 1-8.

6. Soula F, Maria CF, Elisabeth HA, Louise CC. Physical activity participation and cardiovascular fitness in people living with human immunodeficiency virus: a one- year longitudinal study. J AIDS Clinic Res. 2013; 89: 1-5.

7. Santos WR, Paes PP, Santos AP, Machado DRL, Navarro AM, Fernandes APM. Impact of progressive resistance training in Brazilian HIV patients with lipodystrophy. J AIDS Clin Res. 2013; 4: 1-4.

8. Juchem GMV, Lazzarotto AR. Treinamento físico na síndrome lipodistrófica: revisão sistemática. Rev Bras Med Esporte. 2010; 16: 310-13.

9. Brito CJ, Mendes AP, De Paula SO, Nóbrega OT, Córdova C. Impacto do treinamento resistido na força e hipertrofia muscular em HIV-soropositivos. Motriz. 2013; 19: 313-24.

10. Mendes EL, Andaki ACR, Brito CJ, Córdova C, Natali AJ, Amorim RS, et al. Beneficial effects of physical activity in an HIV infected woman with lipodystrophy: a case report. J Med Case Rep. 2011; 5: 1-6.

11. Lindegaard B, Hansen T, Hvid T, Van Hall G, Plomgaard P, Ditleysen S, et al. The effect of strength and endurance training on insulin sensitivity and fat distribution in human immunodeficiency virus-infected patients with lipodystrophy. J Clin Endocrinol Metab. 2008; 93: 3860-69. 
12. Durnin JVGA, Womersley J. Body fat assessed from total body density and its estimation from skinfold thickness: measurements on 481 men and women aged from 16 to 72 years. Br J Nutr. 1974; 32: 77-97.

13. Andrade S, Lan SJJ, Engelson ES, Agin D, Wang J, Heymsfield SB, et al. Use of a DurninWomersley formula to estimate change in subcutaneous fat content in HIV-infected subjects. Am J Clin Nutr. 2002; 75: 587-92.

14. Siri WE. Body composition from fluid space and density. In: Brozek J and Hanschel A. Techniques for measuring body composition. Nat Acad Sci. 1961; 223-4.

15. ISAK. International standards for anthropometric assessment. Sydney: International Society for the Advancement of Kinanthropometry; 2001.

16. Perini TA, Oliveira GL, Ornellas JS, Oliveira FP. Cálculo do erro técnico de medição em antropometria. Rev Bras Med Esporte. 2005; 11: 81-5.

17. Borg G.Psychophysical bases of perceived exertion.J Med Sci Sports Exercise 1982; 14: 377-81.

18. Baechle TR, Earle RW. Essentials of strength training and conditioning. 2nd edition. Ed. Champaign IL: National Strength and Conditioning Association, 2000.

19. American College of Sports Medicine. American College of Sports Medicine position stand. Progression models in resistance training for healthy adults. Med Sci Sports Exerc. 2009; 41: 687-708.

20. Yarasheski KE, Tebas P, Stanerson B, Claxton S, Marin D, Bae K, et al. Resistance exercise training reduces hypertriglyceridemia in $\mathrm{HIV}$-infected men treated with antiviral therapy. $\mathrm{J}$ Appl Physiol. 2001; 90: 133-38.

21. Raso V, Casseb JSR, Duarte AJS, Greve JMDA. Uma breve revisão sobre exercício físico e HIV/AIDS. R Bras Ci Mov. 2007; 15: 115-126.

22. Santos WR, Paes PP, Bueno SMV, Fernandes APM. Exercício físico como tratamento complementar na redistribuição da gordura corporal na síndrome da lipodistrofia em pessoas vivendo com HIV/AIDS. Estudo piloto. EFDeportes.com, Revista Digital 2011; 16.

23. Jones SP,Doran DA, Leatt PB,Maher B,Pirmohamed M. Short-term exercise training improves body composition and hyperlipidaemia in HIV-positive individuals with lipodystrophy. AIDS. 2001; 15: 2049-51.

24. Guerra LMD. Exercício físico, saúde e qualidade de vida em pessoas com o HIV/AIDS em Natal/ Rio Grande do Norte. 2012. Dissertação (Mestrado em Ciências da Saúde) - Programa de Pós-graduação em Ciências da Saúde, Universidade Federal do Rio Grande do Norte, Natal.

25. Roubenoff R, McDermott A, Weiss L, Suri J, Wood M, Bloch R, et al. Short-term progressive resistance training increases strength and lean body mass in adults infected with human immunodeficiency virus. AIDS. 1999; 13: 231-39.

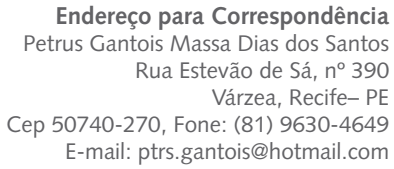

Recebido 03/10/2013

Revisado 05/01/2014

$13 / 01 / 2014$

Aprovado 13/01/2014 